\title{
Use of Future-Oriented Information in User-Centered Product Concept Ideation
}

\author{
Antti Salovaara ${ }^{1}$ and Petri Mannonen ${ }^{2}$ \\ ${ }^{1}$ Helsinki Institute for Information Technology, P.O.Box 9800, 02015 TKK, Finland \\ antti.salovaara@iit.fi \\ ${ }^{2}$ Usability Group, Software Business and Engineering Laboratory, \\ Helsinki University of Technology, P.O.Box 9210, 02015 TKK, Finland \\ petri.mannonenasoberit.hut.fi
}

\begin{abstract}
User-centered product concept design aims at creating concepts of new products. Its success is dependent on the design team's ability to use present-day information to come up with concepts concerning future products. This paper takes as its task to investigate and explore what underlies this use of future-oriented information and what challenges it poses at the creative stages of a design process. The proposed solution is based on an analytic division of available information into (1) trends such as company strategies, trends in the society and working life that denote changing conditions, and (2) stable context features that describe issues that are unlikely to change in the timeframe concerned. A small case study is presented that exemplifies how this analytic distinction can be put into use. More broadly, the paper encourages designers to think reflectively about the nature of information on which design decisions are based.
\end{abstract}

\section{Introduction}

In an innovation management oriented study measuring the number of raw ideas that are needed to make a new commercially successful product, Stevens and Burley found that as many as 3000 unwritten ideas may be needed to end up with a single successful product [23]. Naturally, in such a process much work effort can be wasted. At present, solutions to the problem are often based on lightweight ideation and screening techniques to create ideas easily, and filter out the probable failures as early as possible during the design process.

From a designer point of view, a better alternative would be to improve the percentage of successful ideas. This calls for techniques to guide ideation in more fruitful directions. To address this issue, this paper investigates how future-oriented information, such as company strategies and trends in users' working life and society can be used more effectively in user-centered product concept design (UCPCD).

This paper first presents an outline of UCPCD and analyzes some of the challenges that lie behind the low hit ratio in the current practice. Based on the analysis, the paper provides suggestions for enhanced deployment of future-oriented information to overcome the problems. A report from a case study follows where the usefulness of the hypotheses was explored in a project that developed product concepts for future 


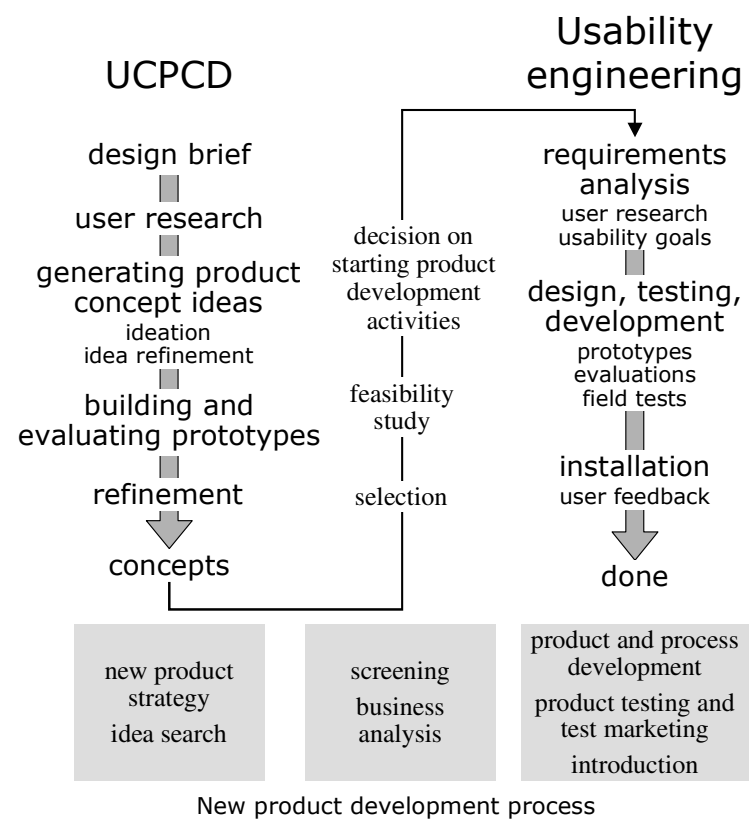

Fig. 1. Relations between user-centered product concept design (UCPCD) and usability engineering, and how they are mapped into a new product development process

maintenance work. This part of the paper is probably most valuable for considerations of contract work, since the focus was on exploratory findings without continuous managerial steering. The paper concludes with evaluation and discussion.

\section{User-Centered Product Concept Design}

Within the field of user-centered design activities, UCPCD is an early-phase exploratory process that aims at creating ideas concerning future products. It has its origins in empathic design [11] and needfinding [22] , which aim to find product ideas by identifying users' needs that are difficult to articulate directly. It is part of the idea search phase in the new product development process [15], and precedes the decision whether the idea is good enough for the actual product development to commence [10]. If the idea is accepted, usability engineering activities (e.g. $[14,17])$ are started.

A simplified relation between UCPCD, usability engineering and new product development is shown in Fig. 1. UCPCD has been originally presented as a process with two iteration cycles of prototyping and testing [7]. In the figure, the latter iteration (using high-fidelity prototypes) has been omitted to stress the nature of UCPCD as a lightweight process, and two sub-phases have been added to idea generation, due to the focus of the paper. Idea screening is also moved outside UCPCD and is assigned to managers who "own" the project, as suggested by Khurana and Rosenthal [10]. The usability engineering process is a simplification of Nielsen's [17] and Mayhew's [14] models. The phases of new product development are from Moore and Pessemier [15]. 


\subsection{Design Phases}

This section provides a quick overview of the standard phases of the concept design process, described briefly in e.g. [19]. Product concept design starts with drafting a design brief - a short description defining what user group and purpose the product should be designed for, and other company-relevant design issues [7]. The contents of the brief vary depending on whether the team is part of the company, an academic research project, or contract work from a consulting firm. The design brief also defines whether the team should aim at developing a concept of a new discontinuous product, or a new incremental version of an existing product.

The design team conducts a user study, often comprising observations and interviews, supplemented with e.g. role-playing, diaries and cultural probes. Ideally, from the material collected the team can identify contextual phenomena and user needs and is able to generate product concepts that answer these issues. Concepts are concretized as interactive prototypes, storyboards or mockups, and are evaluated with users. The ones considered best are selected for further development in a screening process.

\subsection{Challenges in the Process}

As a rather new discipline within HCI, UCPCD is still hampered by many factors that are difficult to control. In product concept idea generation, three challenges can be identified, two relating to ideation, and one to idea refinement. Firstly, after having studied users, ideation may be biased with user data domination, leading to a partial neglect of company-relevant aspects in the design brief. Secondly, although concepts are developed with the future in mind, what the team observes is present-day activities. This can lead to a closed-system assumption: that new forces would not affect user's context in the future, and thus gathered observations would form a reliable basis for design decisions. Studying lead users or other margin segments may alleviate this, but assessing trends in the society and working life should in general play a bigger part in ideation. The problem is addressed in innovation management literature (e.g. [4]) but it is only slowly being distilled into HCI-based concept design.

During idea refinement, weakly grounded contextualization may pose the third challenge. Sketchy ideas need more flesh in the form of e.g. use scenarios. If this contextualization has no linkage to the essentials of the present-day activity, the resulting concepts may be disruptive to users. So, contextualization cannot be independent of how tasks are presently accomplished.

Naturally, UCPCD has other challenges in addition to those already listed. In the discussion, two additional challenges relevant to the paper's topic are discussed.

\subsection{Addressing Challenges by Reflecting on Future-Oriented Information}

The problematization of the standard UCPCD process presented in the previous section is related to how the team reflects upon and uses future-oriented information during the design phases. The connection becomes evident by considering a conceptual division of available information into stable context features (meaning issues that are unlikely to change in the timeframe of interest) and trends (pointing out the differences between present circumstances and their potential trajectories of change). 
The division helps to see which type of information is dominant in the problematic design stages. Stable context features and trends are both future-oriented information, since their division is made based on assumptions about future circumstances.

User data domination during ideation is a result of a narrowly perceived design domain. It could be alleviated by including a wider array of viewpoints to spark creative work. User research observations could be supplemented with company strategies and data on trends in the societal, technological and economic environment. Of the types of future-oriented information, trends seem useful regarding this problem.

Closed-system assumption - another trap during ideation - is also related to the use of trends. Without consideration and explication of what is likely to change in the context, and the team's commitments to such hypotheses, the "creative leap" into the future is not well grounded. Aligning ideas with trends would address the circumstances in the foreseeable future. This can be combined with creative problem-solving methods (such as brainstorming) that are based on an observation that people have difficulties in breaking away from existing solutions and associated restrictions [12]. From the product development point of view, guiding ideation with trends can help focus the innovation towards the foreseeable future without limiting creativity.

The third listed challenge - weakly grounded contextualization - is relevant to the refinement stage where ideas are made more credible and fleshed out. What could be done to avoid disruptive results is to adapt the ideas to present-day features to some necessary extent. The trade-off between the novelty of the concept and conformance to current practice requires balancing. Finding the balance and exploring solutions to other challenges was the target in the case study.

\section{Case Study: Making Concepts for Future Maintenance Work}

The design team explored how to address the challenges in practice in a project where the task was to ideate proactive computing systems for maintenance work. Proactive computing refers to a vision of technology capable of anticipating users' actions and taking initiative, in this way acting on behalf of the user in a beneficial way. Autonomous cooperation between different computing systems and their close connection to real world phenomena are important parts of the vision [24].

This was an academic concept design project where the participating companies did not intervene in the work during ideation. The design brief was explorative: to "develop a description of future practices in maintenance work based on opportunities in proactive computing. The description should help in foreseeing future challenges and in developing tools and machines to be more easily maintainable by service workers, within a time frame of 5 to 10 years."

The two participating companies provide maintenance on elevators and slot machines. In both cases the company is also the manufacturer of the machines maintained. The promise of proactive computing for maintenance work lies in the ongoing trend of equipping machines with increasing communication capabilities. The goal is to enhance machines' capability of event monitoring, self-diagnosis and malfunction communication to service centres via the Internet, which would reduce downtime and help predict errors. However, the same trend also makes it possible to help service workers by providing context-dependent support in maintenance tasks. 


\section{UCPCD}

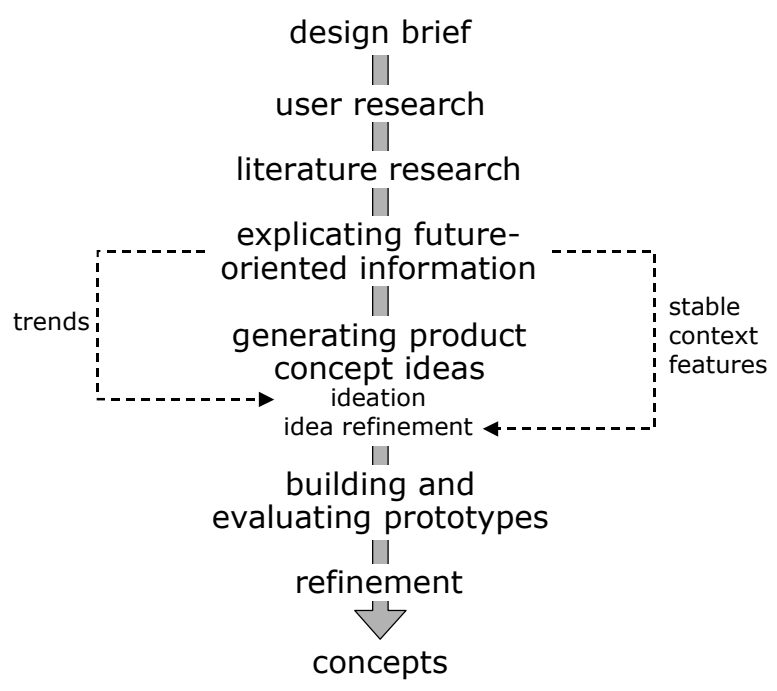

Fig. 2. The user-centered product concept design process, improved with phases for using future-oriented information

\subsection{Planning the Study}

Recognition of the importance of dividing future-oriented information into trends and stable context features was reflected in the methodology of the process. It was noted that the division of information had to take place before starting ideation. Otherwise, if carried out on an on-demand basis, there would be a risk that the team made biased divisions if they had already identified their favourite product concepts after ideation. With early explication, the temptation to interpret data in a post-hoc manner to support favourable conclusions was decreased.

The consideration in the previous section implies that the use of trends precedes stable context features. This means that the team should probably first aim to make a leap into the future during ideation, and then come somewhat back in the time in refinement phase by using presumably stable features in the context.

The overall concept is visualized in Fig. 2. In this figure a separate stage called "Explicating future-oriented information" has been added immediately before the concept generation tasks. From the explication task, information flows lead to the ideation and refinement subtasks. Except for these modifications, the process is similar to the one presented in Fig. 1.

When planning the study, an exploratory approach to methodology on the use of the future-oriented information was chosen. This also serves the purpose of this paper, because rather than proposing a formal model for a design process, it exemplifies the possible uses of the future-oriented information in a reflective manner throughout the design stages. 
However, some decisions were made early on. User research was to be carried out with traditional interviewing and observing methods, to be followed by a literature survey on emerging topics. As the team was familiar with the affinity diagram construction technique, it was chosen as a method in this study and was used during idea refinement. It was known in advance that interactive prototypes would probably be very difficult to build, and therefore the outcome of the project would consist of userevaluated storyboards or passive mock-ups.

\subsection{User Research}

Information about service workers and their context was gathered in two ways: studying the users, and based on the findings, deepening the understanding with literature. The project was started with a study of service workers in both companies. The focus was on learning about the use of present tools, describing the main task categories of maintenance activities, and collecting stories. It was assumed that inquiry into these topics would produce understanding both on procedural aspects of work, as well as on social issues in collaboration and attitudes towards work.

The study covered in all 20 persons from the two companies. Daily work was studied with Contextual Inquiry ( $\mathrm{N}=5)$ [2] and artifact analysis interviews in which workers discussed photographs on their tools $(\mathrm{N}=11)$ [13]. In addition, 4 persons from the companies' R\&D departments were interviewed to obtain a complementary perspective on service work, and to compare their perceptions of work to those of workers themselves. Information was also gained about companies' future strategies.

Analysis consisted of writing aggregated day-in-the-life scenarios (to gather together different observations from Contextual Inquiry), and listing work tasks, workers' opinions and terminology that were collected with different methods. In total, 145 different work tasks, 81 terms and 63 opinions were listed. At this point the importance of a single site visit as a useful unit of analysis was identified, and task sequence models of such site visits were drawn for both companies.

The rationale for these data gathering and analysis methods was their naturalness from the user point of view: interviewing about tools was a natural discussion topic. It was also both economical in terms of the amount of time invested by the team, and supplementary in a way that it revealed issues that were not encountered in Contextual Inquiry. The list creation was easy after day-in-the-life scenario writing and artifact interviews. What the data helped to see were, for instance:

- The intensely mobile nature of work (frequent driving between installed machines)

- The dualistic nature of site visits (either repair or routine maintenance, both of which contain dirty tasks such as cleaning as well as sophisticated tasks such as parameter tuning with a laptop)

- The dualistic nature of customers (requirements coming from users of the machine vs. people who operate the place where the machine is installed)

- The scarcity of the time available to see colleagues (due to the mobility of work)

- Personal service territories as something to be responsible for and proud of.

Interviews with $R \& D$ personnel were helpful in pinpointing topics for literature research, such as questions of changes in expertise requirements of future work, the 
consequences of rearrangements of service territories, or the possibility of implementing proactive computing systems into the existing environment.

In total, there were 8 task categories and 8 general phenomena of such findings. This served as a sufficient introduction to the nature of present-day work, and provided hints concerning future changes in the work context.

\subsection{Literature Research}

Researching the literature on maintenance work was important in providing a second opinion on emerging themes from user research, and in learning about service work in similar work domains, such as photocopier repair. Four topics drew special attention:

- Changing nature of work. This topic emerged both from comments by workers and remarks on technology trends by $\mathrm{R} \& \mathrm{D}$. Investigation into the literature on future of work showed an ongoing debate around the question of de-skilling and skill upgrading: is work becoming automated and simpler or more complicated in the future? There appeared to be no consensus on the trend, but with respect to service work, there appeared to be an incentive to increase hardware modularity and improve he diagnostic capabilities of machines, thus increasing automatization but also sophistication of technology. This drew interest to the following two issues.

- Service work as knowledge work. Skill upgrading is a phenomenon often combined with discussion on knowledge-intensive work. In the observations it became apparent that especially difficult repair tasks had features that resemble knowledge work: creative problem solving under uncertainties, and using information technology frequently to help in such tasks.

- The role of a service worker in the work society. A question was raised also about how workers, customers, and other people in service companies perceive service work. The literature showed workers served two functions: they buffer information between customers and people in the company ("buffers"), and solve maintenance problems for customers ("brokers"). In these roles, technicians are cutpoints in companies' production systems, making companies vulnerable to their loss [1]. As this is not always recognized, tensions tend to form.

- Information sharing among maintenance workers. Orr's ethnographic study on service work and work society [20] pointed out the importance of informal coffee table discussions as an important means for learning. This was also observed in both companies in this study. Such occasions led to a search for further information about knowledge management and the question of horizontal information sharing. This was an interesting issue as both companies had organized their information flow channels to support vertical feedback from workers to managers and R\&D.

The issues from literature provided a basis for explicating the team's assumptions on future working conditions.

\subsection{Explicating Future-Oriented Information}

Having gathered a wealth of domain knowledge, commitments on future-oriented information were explicated before proceeding to concept generation. This information 
consisted of observations on work, workers' opinions, future strategies, information about similar working domains, and discussions found in the literature. Neutrality in moral judgments was found to be important so that trends and features were not selected based on their positive or negative effects on work conditions. Instead, it was within these limits of effects that concepts with positive implications had to be found.

Trends. Section 2.2 suggested trends as a tool to guide ideation into fruitful directions. In the case study, due to the two companies involved, two types of trends were defined. General trends applied to both companies, whereas the rest were companyspecific. Although it was not suggested by the literature, a 24/7 society trend was added because of the visions it could open for future work. In total, the general trends were:

- Increase in surveillance and automatic reporting. Enhanced telecommunication connections with machines and workers' PDAs to maintenance monitoring systems will provide companies with increased scope to monitor the condition of machinesas well as workers' actions. This improves information flow and the ability to anticipate faults, but may also result in more stressful work.

- Increase in evening and night shifts. The 24/7 society phenomenon has a direct effect on service work, since working is easiest during quiet hours, which become scarce if shop opening hours are being extended.

- Proactive maintenance. Technology will support enhanced mechanisms to predict repair needs so that visits are scheduled to take place before faults occur.

- Information outdating and a need for constant updates. Proactive maintenance requires each machine to have individual, tailored maintenance. Proliferation of software components increases individualization of installation configurations. This causes a need to update one's knowledge of the machines more frequently.

The five company-specific trends were mostly inherited from company strategies, and were more focused on specific work issues. We will skip them for the sake of brevity.

Stable Context Features. In the case of finding stable context features in maintenance work within a 5-10-year time frame, explication was done by simplifying task sequence models about typical maintenance visits. With simplification, the level of abstraction was raised, and the model's commitment to present-day factors was decreased, revealing a task structure that summarizes necessary tasks in maintenance visits also in the future: receiving a call-out message, driving to the site, doing routine maintenance or repair, and completing the visit. This model was also in line with company strategies on future work, and did not conflict with the trends identified. The model is shown in Fig. 3.

Task sequence models were chosen as the basis because of the team's user research focus on task categories and tool use. However, a sociogram-like model was also created, describing broker and buffer positions of a worker in relation to workmates, managers and customers. This model originated from literature research findings. 


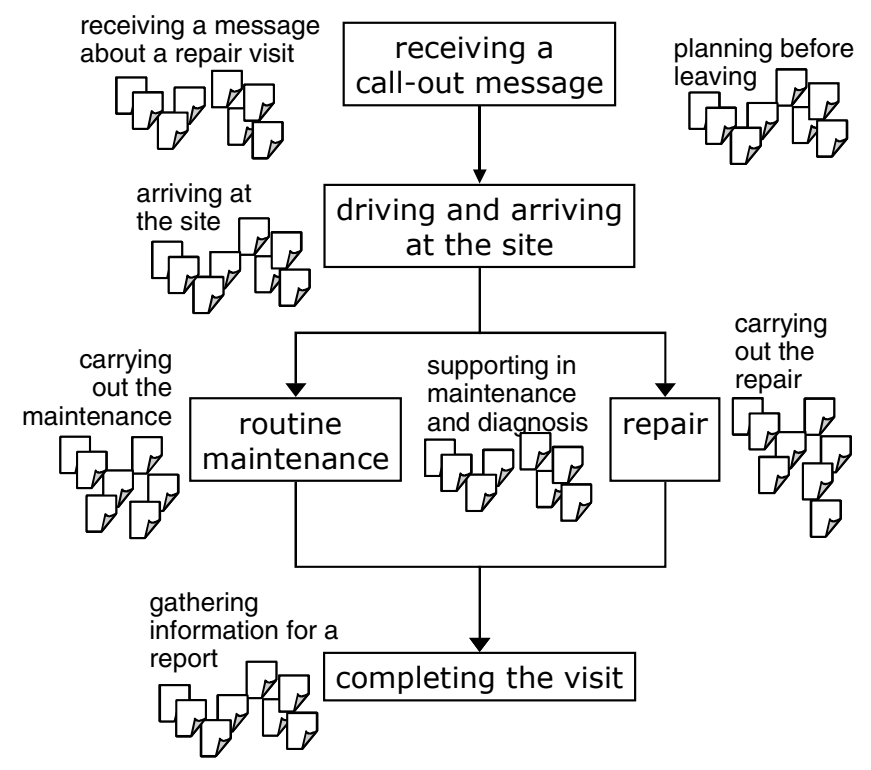

Fig. 3. A schematic visualization a high-level sequence model and its use for providing structure for affinity diagram construction. The clusters of sticker notes denote the main categories of remarks that were identified in the diagram construction process.

\subsection{Ideation Based on Trends}

For a person familiar with the maintenance domain in question, the trends listed are a quick source of ideas about the implications for activities in future work. This was found to be beneficial during ideation. Therefore, instead of envisioning product concepts directly, activities in future work were brainstormed. In order to create a variety of ideas, each trend was considered separately, without any attempt to form a single unified vision. The session was an intensive half-day two-person session where the focus was on inventing activities with positive implications from trends. The persons participating in the session were already familiar with UCPCD, had done project work together in the past, and had participated in the observations and interviews. The rest of the team members did not have as much experience on creative brainstorming and consequently would have probably found this kind of idea generation difficult. Because successful brainstorming is also dependent on the engagement of the people participating (see e.g. [9]), this selection was considered best by the team members. A bigger might possibly have created more ideas, but that is not guaranteed.

Ideas about activities were formulated in one-sentence long microscenarios. Also ideas with difficult implementation were written down if they came up. This was done in order not to rule out any unexpected opportunities that would require a creative leap into the future 5-10 years ahead. Here are three of the resulting 36 microscenarios (with the originating trends in parentheses):

- Serving companies that are only open at night, not during official working hours ("Increase in evening and night shifts"). 
- Repairers start doing their work on a freelance basis when it best fits into their daily schedules ("Increase in evening and night shifts").

- Autonomous reporting of maintenance visits by the machines themselves, with the help of sensors, will relieve the worker from this tedious closing-up task ("Increase in surveillance and automatic reporting").

In a subsequent session, microscenarios were reconsidered in a contemplative session where the whole team was present. With this setup, the microscenarios could be enriched with feedback from all the ethnographers in the project (amounting to four persons in total). Microscenarios were presented one at a time, and PostIt notes were written when ideas came up. This resulted in notes on improved product concept ideas, detailed remarks on design issues, and considerations on future working conditions, amounting to 200 notes in total. Special attention was paid to proactive computing opportunities that would facilitate activities envisioned in microscenarios.

The effect of the two-stage process on the outcome, compared to a straightforward single-stage brainstorming session, is difficult to assess. Trends could have also been cross-tabulated, providing possibly more inspiring starting points for scenario creation. The opportunities to use trends in lightweight idea generation are many, and the methods can be adapted to suit each project's individual requirements.

\subsection{Idea Refinement Based on Stable Context Features}

At this point of the project, a wealth of ideas about proactive computing products had been created in the form of microscenarios and related remarks by team members, but they needed refinement because each idea with its remarks was linked only to the microscenario and the trend from which it had originated. The analysis at the beginning of the paper raised weakly-grounded contextualization as an imminent challenge to idea refinement, and suggested applying stable context features to align ideas with firm contextual data. From previous projects, the team was familiar with affinity diagrams $[2,8]$ as a technique to organize pieces of information into bigger structures. Collecting remarks on PostIt notes in the preceding stage was done with this in mind.

Fig. 3 shows a schematic visualization of the approach taken when refining ideas. A high-level task sequence model of a single site visit was used as a structural backbone to help in organizing the PostIt notes. Each note was placed into its contextually relevant position in the task sequence, and if an idea fitted into multiple positions, the note was duplicated. The organization process resulted in seven note clusters (headings shown in the figure), and gave rise to seven contextualized product concepts. In this stage, care was given to preserve proactive computing opportunities.

\subsection{Evaluation with Users and the Companies}

The refined concept ideas were concretized in storyboard scenarios, and iterated by discussing them with maintenance workers. As a result, two concepts were merged and minor changes were made to each of the concepts. The underlying trends and stable context features were not disclosed to the workers, because they could have been easily interpreted as providing insider information on company strategies. The team felt that it was crucial to avoid any unnecessary misunderstandings. 
To company representatives, full background information was provided. Trends combined with the possibility of proactive computing proved useful in inspiring discussions. As a negative feedback, a bigger emphasis on economic considerations would have been preferred. Although relevant to project success, this feedback does not undermine the validity of this paper's approach, that of the need for explicating trends and stable features. With closer cooperation with companies, such issues could have been included in the future-oriented information and used in idea generation.

\section{Discussion}

The contribution of this paper can be discussed from various viewpoints: are the identified challenges general, and can more challenges be found based on the analytic distinction of future-oriented information? What are the implications of the paper to design practice? Can alternative approaches be suggested to overcome the same challenges? This section addresses these questions.

\subsection{Generalizability of the Identified Challenges}

Predicting the future lies at the heart of any design of future products. By making conscious decisions on the use of available information, the probability of developing successful concepts should be increased. The issues raised in this paper attempt to map out the questions on which such decisions could be based. The generalizability of the contribution can then be partly evaluated by considering whether it helps reveal more of the kind of challenges presented at the beginning of the paper. Indeed, during the study, two new challenges were noted where a division of information into trends and stable features might provide a solution. Both are related to idea refinement.

One is about the risk of inspiration depletion. Coming up with creative ideas requires effort, and doing it repeatedly is difficult and unmotivating. This is, however, the case with unproductive brainstorming results that do not qualify for further development. Inspiration depletion can be overcome if new task-specific information becomes available, thus providing an escape from a fixation to previous problemsolving strategies [6]. In doing this, stable context features can be helpful. As has been seen, user research and literature data can be used to create multiple models. In the case study, the sociogram-like model of buffer and broker relationships could have been used as such an alternative and new viewpoint.

The other challenge is related to internal concept evaluation. Product concepts need to be validated after their generation. But UCPCD is a lightweight process and therefore trials with interactive prototypes and real users cannot always be carried out. There is a need to be able to assess the validity of concepts by other means. With internal evaluation there is a potential problem of within-team evaluation bias, due to the subjective basis of available information. Comparison with identified phenomena and user needs does not qualify, since they have been design drivers for ideation and thus biased for evaluation. Sometimes evaluation is possible by getting feedback from domain experts who are not part of the team, but even before this it would be helpful to be able to do a within-team iteration round. Bias may be decreased with crossvalidation using stable context feature models: one can check if concepts refined with 
one model have conflicts with other models. In the case study, the sociogram would have been such an evaluation basis, since concepts had been refined using the task sequence model. This type of evaluation does not of course replace real evaluations and it requires also that stable models are created before the start of idea generation.

\subsection{Evaluation of the Approach}

As the focus of the paper is essentially on the use of information during the concept design stages, its implications are mostly independent of selected concept design methods or the results of the case study. The case study has merely served as a medium for reflective analysis of the use of available information at different stages of the process. What then needs to be evaluated is the perspective provided by the paper, and the usefulness of its epistemological implications for UCPCD in general.

The implications are of four types. Firstly and most generally, problematization of the use of information in concept design provides a designer with analytical tools that facilitate reflective thinking during the design process. These are the ideas of trends, stable context features, and the described challenges. With these concepts, a designer can assess if false commitments on available information are being made, or if the information on which to base decisions is biased or incomplete.

Secondly, the paper has presented an analytical division of future-oriented information into trends and stable context features. This conceptualization provides more understanding of the nature of information on which concept creation for future products is always based. This helps to choose methods for user and literature research, and also for analysis, in order to distill the right type of information from the context.

Thirdly, by analyzing at what stages each type of future-oriented information is most valuable, the designer can make informed decisions on the methodology to apply when using the information to produce (e.g., brainstorm) good ideas. The transformation of the information and knowledge from user and literature research to the final refinement and evaluation of the concepts can then be streamlined more easily.

Fourthly, the use of trends in guiding ideation into fruitful directions, and giving stable context features in refinement and contextualization, provides an analytical tool for estimating the degree of open-endedness at the concept generation stage. If the focus is on inventing radically new concepts, then the trends can be broad and they can point far into the future, thus widening the design space. If the focus is on minor improvements, the trends can address issues in a 2-3-year time frame, and the stable context features can also describe rather detailed activities.

\subsection{Alternative Approaches to the Challenges}

It is worth considering if alternative approaches exist which provide better solutions for overcoming the listed challenges, and does the use of such approaches exclude the use of the approach presented in this paper. However, this can be done only cursorily.

The case study has three characteristics that need consideration. The first, relating to the project's contract work nature where the companies did not intervene in its progress, has already been mentioned. But there is no reason why defining trends and stable context features should not be possible in within-company projects as well. It may, however, be more challenging to avoid trends which conform too closely to company strategies. Involving outside participants in the explication may be useful. 
The second characteristic is the low involvement of users in the concept generation stages, contrary to the spirit of Participatory Design. While involving users is certainly possible, neutral assessment of future trends may prove problematic. This may result in risk-averse concept development or worker-manager tensions in the team. Therefore, it may be more useful to involve users in the design process when the more traditional development process has started, as shown on the right side of Fig. 1.

The third characteristic is the small number of people involved in the project. Could a bigger team with a multi-disciplinary line-up ensure that no information is misused or forgotten in the process? The answer is that such manning certainly makes it easier to overcome the challenges, but does not conflict with the approach proposed in the paper. Multi-disciplinarity and the explication of future-oriented information can be seen as complementing each other.

Finally, some brief remarks can be made on the techniques applied to overcome individual challenges presented in the paper. These include using scenario methods of futurology (e.g. [3]) to counter user-data domination, or bodystorming [21] to counter within-team evaluation bias. However, these issues must be left for future research.

\section{Conclusions}

The general aim of this paper has been to explore new ways for reflective meta-level thinking during the concept design process, and through analysis to show how that can be beneficial in producing informed ideas about future products. The analysis started by problematizing current approaches and continued with a suggestion to overcome the identified challenges. It was shown to be useful to explicate the gathered information about future use situations with respect to the assumptions made on changes and stable features in the future use context. The suggestion also provided an analysis concerning what stages of a project each type of information would be of most value. A small case study was provided to describe in more detail what the suggestion can imply in practice in terms of epistemological commitments at each level of the process and the methods chosen thereby, especially during ideation and the idea refinement stages. With this treatment, it has been shown how important for any design team it is to consider the nature of information on which the concept design is based, and in this way manage the design space in concept generation.

\section{Acknowledgments}

The authors would like to thank Sirpa Riihiaho and Hannu Kuoppala for their help, and The Finnish Work Environment Fund for funding the project.

\section{References}

1. Barley, S.R.: Technicians in the Workplace: Ethnographic Evidence for Bringing Work into Organization Studies. Administrative Science Quarterly 41(3) (1996) 404-441

2. Beyer, H., Holtzblatt, K.: Contextual Design: Defining Customer-Centered Systems. Morgan Kaufmann, San Francisco (1998) 
3. Coates, J.H.: An Overview of Futures Methods. In: The Knowledge Base of Futures Studies 2, DDM Media Group, Hawthorn Australia (1996) 57-75

4. Craig J., Vogel, C.M.: Creating Breakthrough Products: Innovation from Product Planning to Program Approval. Prentice-Hall PTR, Upper Saddle River (2002)

5. Dahlbäck, N., Jönsson, A., Ahrenberg, L.: Wizard-of-Oz Studies - Why and How. Proc. Intelligent User Interfaces (IUI'93). ACM Press, New York (1993) 193-200

6. Duncker, K. On Problem Solving. Psychological Monograms 270(5) (1945) 1-113

7. Kankainen, A.: UCPCD - User-Centered Product Concept Design. Proc. Designing for User Experiences (DUX'03). ACM Press, New York (2003)

8. Kawakita, J.: The Original KJ Method. Kawakita Research Insitute, Tokyo (1982)

9. Kelley, T., Littman, J.: The Art of Innovation. Doubleday, New York (2001)

10. Khurana, A., Rosenthal, S.R.: Towards Holistic "Front Ends" in New Product Development. Journal of Product Innovation Management 15(1) (1998) 57-74

11. Leonard, D., Rayport, J.F.: Spark Innovation through Empathic Design. Harvard Business Review 75(6) (1997) 102-113

12. Lumsdaine, E., Lumsdaine M.: Creative Problem Solving: Thinking Skills for a Changing World. McGraw-Hill, New York (1995)

13. Mannonen, P., Kuoppala, H., Nieminen, M.P.: Photograph-Based Artefact Analysis. Proc. Interact'03. IOS Press, Amsterdam (2003) 833-836

14. Mayhew, D.J.: The Usability Engineering Lifecycle. Morgan Kaufmann, San Francisco (1999)

15. Moore, W.L., Pessemier, E.A.: Product Planning and Management: Designing and Delivering Value. McGraw-Hill, New York (1993)

16. National Research Council: The Changing Nature of Work: Implications for Occupational Analysis. National Academies Press, Washington (1999)

17. Nielsen, J.: Usability Engineering. Academic Press, Boston (1993)

18. Nielsen, J.: Scenarios in Discount Usability Engineering. In Carroll, J.M. (ed.) ScenarioBased Design: Envisioning Work and Technology in System Development. John Wiley \& Sons, New York (1995)

19. Nieminen, M., Mannonen, P., Turkki, L.: User-Centered Concept Development Process for Emerging Technologies. Proc. NordiCHI'04. ACM Press, New York (2004) 225-228

20. Orr, J.E.: Talking about Machines: An Ethnography of a Modern Job. ILR Press, Ithaca (1996)

21. Oulasvirta, A., Kurvinen, E., Kankainen, T.: Understanding Contexts by Being There: Case Studies in Bodystorming. Personal and Ubiquitous Computing 7(2) (2003) 125-134

22. Patnaik, D., Becker R.: Needfinding: The Why and How of Uncovering People's Needs. Design Management Journal 10(2) (1999) 37-43

23. Stevens, G.A., Burley, J.: 3,000 raw ideas $=1$ commercial success! Research Technology Management 44(3) (1997) 16-27

24. Tennenhouse, D.: Proactive Computing. Communications of the ACM 43(5) (2000) 43-50. 